\title{
Hypernatremia is common in patients with severe COVID-19 and indicates a poor prognosis
}

\section{Anna Sjöström ( $\nabla$ anna.sjostrom@ki.se )}

Karolinska Institutet

\section{Susanne Rysz}

Karolinska Institutet

\section{Henrik Sjöström}

Karolinska institutet

Charlotte Höybye

Karolinska Institutet

\section{Research Article}

Keywords: COVID-19, SARS-Cov-2, Electrolytes, Fluid balance, Aldosterone, AVP

Posted Date: September 23rd, 2020

DOl: https://doi.org/10.21203/rs.3.rs-81965/v1

License: @ (i) This work is licensed under a Creative Commons Attribution 4.0 International License. Read Full License 


\section{Abstract}

Background: Fluid homeostasis, including electrolyte balance, is dependent on an interaction between the renin-angiotensin-aldosterone system (RAAS) and the release of arginine vasopressin from the pituitary. An imbalance can lead to both hypo- and hypernatremia.

We investigated the frequency, dynamics and severity of electrolyte imbalance in critically ill patients with COVID-19.

Methods: In this retrospective cohort study 223 patients with confirmed COVID-19, treated at the intensive care unit (ICU), were included. Levels of electrolytes, base excess, $\mathrm{pH}$ and serum osmolality were collected from the laboratory database. Clinical data was retrieved from patients' medical records.

Results: Hyponatremia was present in $63 \%$ of the patients, at admission. Within two weeks of hospitalization, $65 \%$ of the patients developed hypernatremia often combined with a rise of base excess. The mortality rate was twice as high in the group with hypernatremia compared to the patients not developing hypernatremia. Treatment of hypertension before the onset of COVID-19 was more common in patients without hypernatremia.

Conclusion: Our study shows that hypernatremia is very common in severe COVID-19. Patients that developed hypernatremia needed longer time in ICU and had a higher risk of dying, suggesting that the level of sodium is an important indicator of severity in COVID-19.

\section{Introduction}

Already in the early stages of the pandemic, the renin-angiotensin-aldosterone system (RAAS) have been in focus due to the virus, SARS-CoV-2, enters the body through the angiotensin converting enzyme (ACE)receptors, ACE2(1), that are widely expressed in the human body. Increased levels of angiotensin II have been found in patients with severe COVID-19 and "loss of brake" by inhibiting ACE2 have been discussed as part of the pathophysiology of severe disease. Overactivation of RAAS leads to high levels of angiotensin II stimulating release of aldosterone from the adrenal gland followed by increased release of antidiuretic hormone, arginine vasopressin (AVP), from the pituitary. AVP can also be released by low blood volume and high serum osmolality. Increased levels of aldosterone may cause hypertension, hypernatremia, hypokalemia and metabolic alkalosis. High levels of AVP, on the other hand, results in hyponatremia. When this overactivation of RAAS occurs, and which exact physiological consequences this leads to, is not fully known in COVID-19. This metabolic component by disturbance of RAAS, in combination with the known respiratory failure in COVID-19, makes the electrolyte- and acid-base patterns unpredictable. 
There are several reports of hyponatremia, at admission, in COVID-19 patients indicating that the level of hyponatremia could be a risk factor of severe disease and mortality (2-4). Hyponatremia in severely ill patients in the intensive care unit (ICU) is a known complication to somatic diseases such as pneumonia and heart failure (5) but risk factors of developing hyponatremia in COVID-19 patients have not been explored. Syndrome of inappropriate secretion of antidiuretic hormone (SIADH) is a common cause of hyponatremia in viral pneumonia and there are several case reports of this neuroendocrinological disturbance in COVID-19 patients (6-10). In SIADH there is release of AVP from the back lobe of the pituitary despite normal or low serum osmolality. Increased AVP leads to hyponatremia due to excessive inflow of water through aquaporins in the proximal tubules of the kidney (11).

A recent publication, of a small cohort of patients with severe COVID-19, indicates the importance of therapy resistant hypernatremia which prolonged the total hospital stay (12). The effect of hypernatremia on mortality risk, in COVID-19, is still not elucidated. Hypernatremia can be a serious condition associated with longer hospital stays and increased mortality(13). It is not uncommon with hypernatremia in the ICU and studies have reported a frequency of $4,3-15,8 \%$ depending on type of ICU (medical/surgical) and the definition of hypernatremia $145-150 \mathrm{mmol} / \mathrm{L}$ (14-20). The causes of ICU-related hypernatremia are often assessed as iatrogenic due to saline infusions and the patient's loss of autonomy and inability to drink (15). Complications associated with hypernatremia and hyperosmolality include dehydration, hyperventilation, muscle cramps and rhabdomyolysis, brain dehydration and shrinkage (21).

Low levels of potassium at admission has been associated with poor prognosis and severe disease but little is known of the dynamics over time $(22,23)$. Chloride homeostasis has been briefly discussed in previous studies without finding any prognostic value at admission (24).

The frequently reported deviations in electrolytes during COVID-19 might affect treatment, length of hospital stay and mortality. Patients with severe COVID-19 are often hospitalized over a long time period and further characterization of electrolyte levels over time is therefore of great interest. Here we show the dynamic pattern of electrolytes, and acid-base biomarkers, measured repeatedly in critically ill patients with documented COVID-19 in the ICU.

\section{Aim/Objectives:}


The primary aim was to characterize the dynamics of plasma electrolytes, and acid-base biomarkers, during the first two weeks of severe COVID-19.

Secondary aims were to examine the value of sodium as a prognostic marker for length of hospitalization, acute kidney injury (AKI) and mortality. Electrolyte levels together with base excess and $\mathrm{pH}$, were also evaluated for characteristics of SIADH, dehydration/diabetes insipidus or hyperaldosteronism.

\section{Results}

Our cohort of ICU patients was divided into two main groups, depending on development of hypernatremia or not. Dynamics of sodium in both groups followed the same pattern with hyponatremia at admission and slowly increasing levels of sodium over the first weeks of hospitalization.

Hyponatremia at admission was seen in $63 \%$ of the patients. Over time, hypernatremia developed in 146 patients $(65 \%)$ and 53 patients (24\% of the total cohort) had peak levels of $150 \mathrm{mmol} / \mathrm{L}$ or above. Over the two-week study period 173 patients $(78 \%)$ had at least one sodium result below $137 \mathrm{mmol} / \mathrm{L}$.

Median age of the cohort was 59 years, the majority were males (79\%), median BMI was $29.4 \mathrm{~kg} / \mathrm{m} 2,30 \%$ had diabetes type 2 and the most common comorbidity was hypertension (40\%) (Table 1). One of the patients had diabetes type 1 .

Table 1: Descriptive data of 223 critically ill patients with COVID-19 


\begin{tabular}{|c|c|c|c|c|c|}
\hline & All & $\begin{array}{l}\text { Non- } \\
\text { hypernatremia } \\
(<145 \mathrm{mmol} / \mathrm{L})\end{array}$ & $\begin{array}{l}\text { Moderate } \\
\text { Hypernatremia } 145- \\
149 \text { mmol/L }\end{array}$ & $\begin{array}{l}\text { Severe } \\
\text { Hypernatremia } \\
>150 \mathrm{mmol} / \mathrm{L}\end{array}$ & $p$ \\
\hline Patients, N (\%) & 223 & 77 (35\%) & 93 (42\%) & $53(24 \%)$ & \\
\hline $\begin{array}{l}\text { Median age, } \\
\text { (IQR) }\end{array}$ & 59 & $59(50-64)$ & $59(52-64)$ & $57(51-65)$ & $p=0.862$ \\
\hline Male sex, N (\%) & 177 & $62(81 \%)$ & $71(76 \%)$ & 44 (83\%) & $p=0.603$ \\
\hline $\begin{array}{l}\text { Median BMI, } \\
\text { (IQR) }\end{array}$ & $\begin{array}{l}29.4 \\
(26.5- \\
32.5)\end{array}$ & $29,3(26.2-32.6)$ & $29.8(26.9-32.8)$ & $\begin{array}{l}28.7(26.3- \\
30.9)\end{array}$ & $p=0.238$ \\
\hline $\begin{array}{l}\text { Hypertension, } \\
\mathrm{N}(\%)\end{array}$ & $\begin{array}{l}89 \\
(40 \%)\end{array}$ & 38 (49\%) & 37 (40\%) & $14(26 \%)$ & $p=0.032$ \\
\hline $\begin{array}{l}\text { Diabetes } \\
\text { mellitus type } 2 \text {, } \\
\text { N (\%) }\end{array}$ & $\begin{array}{l}66 \\
(30 \%)\end{array}$ & $23(30 \%)$ & 25 (27\%) & 18 (34\%) & $p=0.665$ \\
\hline OPD, N (\%) & $\begin{array}{l}40 \\
(18 \%)\end{array}$ & 15 (19\%) & $19(20 \%)$ & $6(11 \%)$ & $p=0.351$ \\
\hline
\end{tabular}

During the study period, $86 \%$ of the patients received mechanical ventilation, $44 \%$ developed $A K I$ and $30 \%$ did not survive (Table 2). Median follow up time was 56 days for the non-hypernatremia group and 55 days for the hypernatremia group. Total accumulated follow up time was 10534 days.

Table 2: Outcome data of 223 critically ill patients with COVID-19. 


\begin{tabular}{lllll} 
& All & $\begin{array}{l}\text { Non-hypernatremia } \\
(<145 \mathrm{mmol} / \mathrm{L})\end{array}$ & $\begin{array}{c}\text { Hypernatremia } 145- \\
149 \mathrm{mmol} / \mathrm{L}\end{array}$ & $\begin{array}{l}\text { Hypernatremia } \\
>150 \mathrm{mmol} / \mathrm{L}\end{array}$ \\
\hline $\begin{array}{l}\text { Need of mechanical } \\
\text { ventilation, N (\%) }\end{array}$ & $\begin{array}{l}191 \\
(86 \%)\end{array}$ & $47(61 \%)$ & $91(98 \%)$ & $53(100 \%)$ \\
\hline $\begin{array}{l}\text { Median time on } \\
\text { ventilator }\end{array}$ & $\begin{array}{l}11 \\
\text { days }\end{array}$ & 3 days & 14 days & 15 days \\
\hline Median time in ICU & $\begin{array}{l}14 \\
\text { days }\end{array}$ & 8 days & 16 days & 17 days \\
\hline Median hospital stay & $\begin{array}{l}22 \\
\text { days }\end{array}$ & 18 days & 25 days & 27 days \\
\hline $\begin{array}{l}\text { Acute kidney injury, N } \\
(\%)\end{array}$ & $\begin{array}{l}98 \\
(44 \%)\end{array}$ & $24(31 \%)$ & $47(51 \%)$ & $9(17 \%)$ \\
\hline Need of dialysis, N (\%) & $\begin{array}{l}51 \\
(23 \%)\end{array}$ & $17(22 \%)$ & $25(27 \%)$ & $16(30 \%)$ \\
\hline Mortality, N (\%) & $\begin{array}{l}66 \\
(30 \%)\end{array}$ & $16(21 \%)$ & $34(37 \%)$ &
\end{tabular}

\section{Demographics of comorbidities, age and gender in hypernatremia.}

There were no differences in age $(p=0.685)$, gender $(p=0.758)$, DM II $(p=0.948)$ or OPD $(p=0.663)$ between the group with moderate hypernatremia, the group with severe hypernatremia or the group without. There was a smaller proportion of patients with hypertension in the group with hypernatremia ( $p$ $=0.037)$.

\section{Relationships between hypernatremia, time in hospital, time in the ICU and the need of mechanical ventilation.}

The presence of hypernatremia was associated with a longer time in hospital $(p=0.006$, Mann-Whitney U-test), with a median hospital stay of 24 days in the hypernatremia group and 18 days in the nonhypernatremia group. Hypernatremia was similarly associated with a longer time in the ICU $(p<0.001$, tested by Mann-Whitney U-test) with a median ICU stay of 16 days in the hypernatremia group compared to 8 days in the non-hypernatremia group. The need for ventilatory support was seen in a larger 
proportion ( $p<0.001$, Pearson's $\chi^{2}$ test) of the hypernatremia group $(144 / 146,99 \%)$ compared to the nonhypernatremia group $(47 / 77,61 \%)$.

\section{Effects of hypernatremia on the likelihood of death.}

To investigate the effect of hypernatremia on the likelihood of death, a binary logistic regression was conducted. Age, gender and presence of hypernatremia, diabetes mellitus type 2, hypertension and OPD were entered into the model. Linearity of the continuous variable with respect to the logit of the dependent variable was assessed via the Box-Tidwell procedure. The logistic regression model was statistically significant $\left(c^{2}(6)=28.753, p<0.001\right)$. Three of the individual variables were statistically significant; age, gender and the presence of hypernatremia. An increase in age by one year was associated with 1.063 times higher odds of death, and males had 2.934 times higher odds of death compared to females. Presence of hypernatremia was associated with 2.051 times higher odds of death.

\section{Laboratory parameter dynamics over time}

The dynamics of the laboratory parameters for the whole cohort is presented in Figure 1. For analysis of laboratory parameter dynamics over time, the group with hypernatremia was further divided into two subgroups; one with moderate hypernatremia (145-149 mmol/L) and one with severe hypernatremia (>149 mmol/L). ANOVA analysis was performed to investigate significant differences between the three groups in laboratory parameters day $0,4,8$ and 12 . The different patterns of laboratory parameters between the 3 groups are presented in Figure 2. Day 0 no differences between the groups were seen in any of the 5 parameters investigated (sodium, potassium, chloride, $\mathrm{pH}$ and base excess). On day 4 only differences between sodium levels could be shown. At day 8 , additional to differences in sodium levels, there were a significant difference in chloride levels between the moderate hypernatremia group and the severe hypernatremia group as well as significant differences in base excess between the patients with no hypernatremia and the other two groups. Day 12, group ANOVA showed significant differences in all parameters except potassium. The no hypernatremia group was different from all the other groups and there were significant differences between moderate and severe regarding sodium and chloride. Potassium levels showed significant differences between the no hypernatremia and moderate hypernatremia groups, but the group ANOVA was not significant $(p=0.052)$. For more information of group differences and $p$-values see additional table 3 [Additional file 3].

The sub-group with no hypernatremia had a median picture of initial hyponatremia and respiratory alkalosis with levels of chloride and potassium in the lower range of normal. Over time pH slowly 
decreased and the other parameters were kept in the normal range.

On admission, the moderate hypernatremia group also had a median picture of hyponatremia, respiratory alkalosis and levels of chloride and potassium in the lower range of normal. Shortly after admission $\mathrm{pH}$ dropped to normal levels and BE increased together with chloride to end up in metabolic alkalosis after 710 days.

Finally, in the group of patients with severe hypernatremia the dynamics was resembling the moderate hypernatremia group. Initially displaying a pattern similar to the other groups, with hyponatremia and respiratory alkalosis. Compared to the moderate hypernatremia group, the severe hypernatremia group developed a more pronounced metabolic, hyperchloremic alkalosis.

\section{Discussion}

In this study we found that during the first two weeks of hospitalization $65 \%$ of 223 patients with severe COVID-19 developed sodium levels above $144 \mathrm{mmol} / \mathrm{L}$ and thus hypernatremia. We also found that patients with hypernatremia needed twice as long stay in ICU and had a higher risk of dying than patients without hypernatremia.

It cannot be excluded that the very high rate of hypernatremia we found could be iatrogenic and/or caused by activation of several endogenous regulating systems. The biochemical electrolyte shifts due to slight dehydration following treatment according to the acute respiratory distress syndrome (ARDS)treatment protocol together with the virus activation of the RAAS would be expected to induce an increased reabsorption of sodium and an increased release of AVP. The latter could over time diminish the amount of available AVP and as a consequence decrease the reabsorption of water in the kidney, diabetes insipidus possibly in partial and transient form. In the present cohort, all patients with sodium levels above $149 \mathrm{mmol} / \mathrm{L}$ received active sodium-lowering treatment. Therefore, the very high sodium levels were unintended and likely caused by the disease.

The potential over-activation of RAAS with elevated angiotensin II levels could induce hyperaldosteronism. Aldosterone acts on the $\mathrm{Na} / \mathrm{K}$ channels in the kidneys distal tubule mediating increased influx of sodium and outflow of potassium together with hydrogen ions. This results in hypertension, hypernatremia, hypokalemia and metabolic alkalosis. 
Over time many patients in the study developed metabolic alkalosis with elevated $\mathrm{pH}$ and $\mathrm{BE}$. Augmentation of $\mathrm{BE}$ is often a compensatory mechanism in respiratory acidosis, the body's way of increasing low $\mathrm{pH}$ due to respiratory failure. In this cohort, though, the $\mathrm{pH}$ was alkalotic at admission. This respiratory alkalosis is suspected to be a result of the silent hypoxia, without hypercapnia, caused by the viral pneumonia.

Within a few days' pH decreased to normal range in the hypernatremia patients but much slower in the non-hypernatremia group. The rise of BE did start at admission, in all groups, peaking days 7-11. This metabolic alkalosis was typed as chloride resistant, even hyperchloremic, also indicating possible aldosterone and dehydration/AVP involvement.

Of note, more patients in the subgroup who did not develop hypernatremia were receiving treatment for hypertension before the onset of COVID-19 and it can be speculated if the development of hypernatremia was inhibited by the anti-hypertensive drugs. In addition, the length of hospital stay was longer in the hypernatremia group and the mortality was higher compared to the non-hypernatremia group. Altogether, these clinical observations suggest that hypernatremia is an indicator of severe illness.

There was no difference in level of hyponatremia at admission between intubated and non-intubated. In the group of not intubated patients only one patient had sodium levels above $144 \mathrm{mmol} / \mathrm{L}$. If this finding was due to less severe disease of the non-intubated patients or that the sedation, intubation and/or ventilation itself increases the risk of hypernatremia is an unanswered issue in this study.

A well-known cause of hyponatremia is SIADH where increased release of AVP elicits excess water reabsorption from aquaporins in the kidney's tubule. Our study shows an incidence of hyponatremia of $63 \%$ at admission. The main reason of admittance was atypical viral pneumonia with respiratory failure and, in this patient group, SIADH is known to be one of the most common underlying causes of hyponatremia.

In theory, patients presenting with hyponatremia at admission caused by SIADH could be of higher risk of developing severe hypernatremia later due to depletion of available AVP. This is difficult to substantiate due to patients seeking medical care at different time points and possible individual variation in AVP storage pool.

Synthetic AVP (desmopressin) is used as procoagulant treatment, e.g. in von Willebrand's disease (vWD), as it increases platelet release of coagulation Factor VIII and von Willebrand Factor after administration. At admission the patients presented with low levels of sodium and suspected SIADH. The high release of AVP could be a part of the procoagulant state of COVID-19 further impaired by hyperosmolality which 
also increases the risk of venous thromboembolism(25). In our study very high levels of serum osmolality was seen a few days after admission, when sodium increased and the level of AVP released might have become insufficient.

There are some limitations to this study. Missing data is a hazard for retrospective studies and patients are not sampled according to a specific protocol leading to some variations in timing of blood tests between the patients. This study was based on routine blood tests and unfortunately no hormone biomarkers like aldosterone, angiotensin II or renin were analyzed. It would have been very interesting to see hormone results and also levels of sodium, potassium and osmolality in urine. This study, however, represents a large clinical consecutively recruited cohort of ICU patients, adding to the generalizability of our results.

Potassium infusions made it difficult to assess the untreated levels of hypokalemia present.

Another issue is that hemolysis is not registered by the machines used for blood gas analysis to the same extent and way as routine biochemistry lab does which potentially lead to a risk of falsely elevated potassium levels. The presence of CRRT is also a potential source of error due to its effect on electrolyte levels.

The presence or extent of hypertension during in-patient care was inaccessible owing to the majority of the patients receiving sedative drugs due to being intubated and mechanically ventilated.

\section{Conclusion}

In this retrospective study we studied a heterogeneous group of critically ill patients who received a multitude of intensive care and medications. Several factors and regulatory systems were involved to establish homeostasis and improve their condition. However, based on our findings with a high number of patients with hyponatremia at admission followed by hypernatremia the following weeks it can be speculated if this pattern of sodium could be a result of an over-activation of RAAS leading to a transient insufficiency of AVP. This would also be in line with the effect of SARS-cov2 on the ACE2 receptor. Furthermore, patients with hypernatremia displayed a more severe course of COVID-19 and the mortality rate was higher in this group compared to the patients not developing hypernatremia. To further understand the variations in sodium possible treatments, prospective studies with structured 
measurements of serum levels of angiotensin II, aldosterone, AVP, copeptin, urinary sodium, potassium and osmolality would be of great value.

\section{Materials And Methods}

The study was a retrospective observational cohort study of 245 ICU patients with COVID-19 admitted to Karolinska University Hospital (KUH), March 5 to April 22, 2020. The study was approved by The Swedish Ethical Review Authority (N 2020-01752).

Inclusion criteria were positive RT PCR for SARS-Cov2, admission to a hospital connected to the KUH laboratory and need of ICU care. Patients were, by reviewing medical records, until discharged from the hospital or deceased. Last day of follow-up was June 30, 2020.

Patients transferred to a hospital in another region, before they recovered, were excluded.

Descriptive data on age, gender, body mass index (BMI), comorbidities (medically treated diabetes mellitus type 2 (DM II), hypertension and obstructive pulmonary disease (OPD)) was retrieved together with information on day of hospitalization and admittance to ICU, need of intubation and length of mechanical ventilation, presence of AKI and continuous renal replacement therapy (CRRT), death and total hospital stay.

\section{Outcomes:}

Patients were defined as discharged when dismissed from inpatient care to their homes or to a rehabilitation facility. Information on mortality was retrieved from the medical records from admittance to hospital until last day of follow up.

AKI was classified as present when noted in the medical records or by ICD code.

Patients were primarily differentiated into two groups with either hypernatremia or no hypernatremia. According to the patients' peak level of sodium three sub-groups were further identified; no hypernatremia ( $<145 \mathrm{mmol} / \mathrm{L})$, moderate hypernatremia $(145-149 \mathrm{mmol} / \mathrm{L})$ and severe hypernatremia $(>149 \mathrm{mmol} / \mathrm{L})$,

Laboratory parameters were collected from the KUHs laboratory database. Information on levels of plasma sodium, potassium and serum osmolality as well as blood gas analysis of arterial chloride, base excess $(\mathrm{BE})$ and $\mathrm{pH}$ was recorded from day of admittance and onwards, when measured, daily until 2 weeks had passed. If there were several measurements of a parameter the same day, the result closest to 
8.00 AM was recorded. For more information of laboratory equipment and methods see additional table 1 [Additional file 1].

Study size was finalized at 223 patients after exclusion of 22 patients due to lost to of follow-up. The number of patients with relevant blood tests at the respective time points are shown in an additional table [Additional file 2].

\section{Statistical analysis:}

Statistical analysis was performed using Excel (Microsoft office 365, Microsoft Corp., Redmond, WA, USA) and SPSS (version 26.0, IBM Corp., Armonk, NY, USA). Normality of data was assessed using Shapiro-Wilk test. Pearson's $\chi^{2}$ test was used to evaluate possible differences in distributions between groups. Binary logistic regression was used to investigate the effect of clinical variables on the development of hypernatremia and on the risk of death. Medians at each time point were calculated from existing laboratory results and no interpolation of data was performed. A p-value $<0.05$ was considered significant.

\section{References}

1. Samavati L, Uhal BD. ACE2, Much More Than Just a Receptor for SARS-COV-2. Front Cell Infect Microbiol. 2020;10:317.

2. Berni A, Malandrino D, Parenti G, Maggi M, Poggesi L, Peri A. Hyponatremia, IL-6, and SARS-CoV-2 (COVID-19) infection: may all fit together? J Endocrinol Invest. 2020.

3. Aggarwal S, Garcia-Telles N, Aggarwal G, Lavie C, Lippi G, Henry BM. Clinical features, laboratory characteristics, and outcomes of patients hospitalized with coronavirus disease 2019 (COVID-19): Early report from the United States. Diagnosis (Berl). 2020;7(2):91-6. 
4. Wu Y, Hou B, Liu J, Chen Y, Zhong P. Risk Factors Associated With Long-Term Hospitalization in Patients With COVID-19: A Single-Centered, Retrospective Study. Front Med (Lausanne). 2020;7:315.

5. Zilberberg MD, Exuzides A, Spalding J, Foreman A, Jones AG, Colby C, et al. Epidemiology, clinical and economic outcomes of admission hyponatremia among hospitalized patients. Curr Med Res Opin. 2008;24(6):1601-8.

6. Gemcioglu E, Karabuga B, Ercan A, Erden A. A case of Inappropriate Antidiuretic Hormone Secretion Syndrome Associated with COVID-19 Pneumonia. Acta Endocrinol (Buchar). 2020;16(1):110-1.

7. De La Flor Merino JC, Mola Reyes L, Linares Gravalos T, Roel Conde A, Rodeles Del Pozo M. [An unusual case of severe acute hyponatremia in patient with COVID-19 infection]. Nefrologia. 2020;40(3):356-8.

8. Habib MB, Sardar S, Sajid J. Acute symptomatic hyponatremia in setting of SIADH as an isolated presentation of COVID-19. IDCases. 2020;21:e00859.

9. Tantisattamo E, Reddy UG, Duong DK, Ferrey AJ, Ichii H, Dafoe DC, et al. Hyponatremia: A Possible Immuno-Neuroendocrine Interface with COVID-19 in a Kidney Transplant Recipient. Transpl Infect Dis. 2020:e13355.

10. Ho KS, Narasimhan B, Kumar A, Flynn E, Salonia J, El-Hachem K, et al. Syndrome of inappropriate antidiuretic hormone as the initial presentation of COVID-19: A novel case report. Nefrologia. 2020.

11. Ellison DH, Berl T. Clinical practice. The syndrome of inappropriate antidiuresis. N Engl J Med. 2007;356(20):2064-72.

12. Zimmer MA, Zink AK, Weisser CW, Vogt $U$, Michelsen A, Priebe HJ, et al. Hypernatremia-A Manifestation of COVID-19: A Case Series. A A Pract. 2020;14(9):e01295.

13. Waite MD, Fuhrman SA, Badawi O, Zuckerman IH, Franey CS. Intensive care unit-acquired hypernatremia is an independent predictor of increased mortality and length of stay. $\mathrm{J}$ Crit Care. 2013;28(4):405-12.

14. Darmon M, Timsit JF, Francais A, Nguile-Makao M, Adrie C, Cohen Y, et al. Association between hypernatraemia acquired in the ICU and mortality: a cohort study. Nephrol Dial Transplant. 2010;25(8):2510-5.

15. Lindner G. "Hypernatremia in the intensive care unit-an iatrogenic complication?". J Crit Care. 2013;28(2):214-5.

16. Lindner G, Funk GC, Lassnigg A, Mouhieddine M, Ahmad SA, Schwarz C, et al. Intensive care-acquired hypernatremia after major cardiothoracic surgery is associated with increased mortality. Intensive Care Med. 2010;36(10):1718-23.

17. Lindner G, Funk GC, Schwarz C, Kneidinger N, Kaider A, Schneeweiss B, et al. Hypernatremia in the critically ill is an independent risk factor for mortality. Am J Kidney Dis. 2007;50(6):952-7.

18. O'Donoghue SD, Dulhunty JM, Bandeshe HK, Senthuran S, Gowardman JR. Acquired hypernatraemia is an independent predictor of mortality in critically ill patients. Anaesthesia. 2009;64(5):514-20. 
19. Polderman KH, Schreuder WO, Strack van Schijndel RJ, Thijs LG. Hypernatremia in the intensive care unit: an indicator of quality of care? Crit Care Med. 1999;27(6):1105-8.

20. Vandergheynst F, Sakr Y, Felleiter P, Hering R, Groeneveld J, Vanhems P, et al. Incidence and prognosis of dysnatraemia in critically ill patients: analysis of a large prevalence study. Eur J Clin Invest. 2013;43(9):933-48.

21. Lindner G, Funk GC. Hypernatremia in critically ill patients. J Crit Care. 2013;28(2):216 e11-20.

22. Chen D, Li X, Song Q, Hu C, Su F, Dai J, et al. Assessment of Hypokalemia and Clinical Characteristics in Patients With Coronavirus Disease 2019 in Wenzhou, China. JAMA Netw Open. 2020;3(6):e2011122.

23. Wendel Garcia PD, Fumeaux T, Guerci P, Heuberger DM, Montomoli J, Roche-Campo F, et al. Prognostic factors associated with mortality risk and disease progression in 639 critically ill patients with COVID-19 in Europe: Initial report of the international RISC-19-ICU prospective observational cohort. EClinicalMedicine. 2020;25:100449.

24. Lippi G, South AM, Henry BM. Electrolyte imbalances in patients with severe coronavirus disease 2019 (COVID-19). Ann Clin Biochem. 2020;57(3):262-5.

25. Keenan CR, Murin S, White RH. High risk for venous thromboembolism in diabetics with hyperosmolar state: comparison with other acute medical illnesses. J Thromb Haemost. 2007;5(6):1185-90.

\section{Declarations}

Acknowledgments

We are grateful to all the nurses and doctors, who worked day and night, caring for these severely sick patients and making it possible for us to conduct this study

\section{Author contributions}

AS designed the study, collected, analyzed and interpreted the data, wrote the first draft and revised the manuscript. SR has interpreted the data and revised the manuscript. HS analyzed and interpreted the data and revised the manuscript. $\mathrm{CH}$ has supervised the course of the study, interpreted the data and revised the manuscript. All authors read and approved the final manuscript 
Additional information:

Competing interests and disclosure

The authors declare no competing financial interests.

Figures
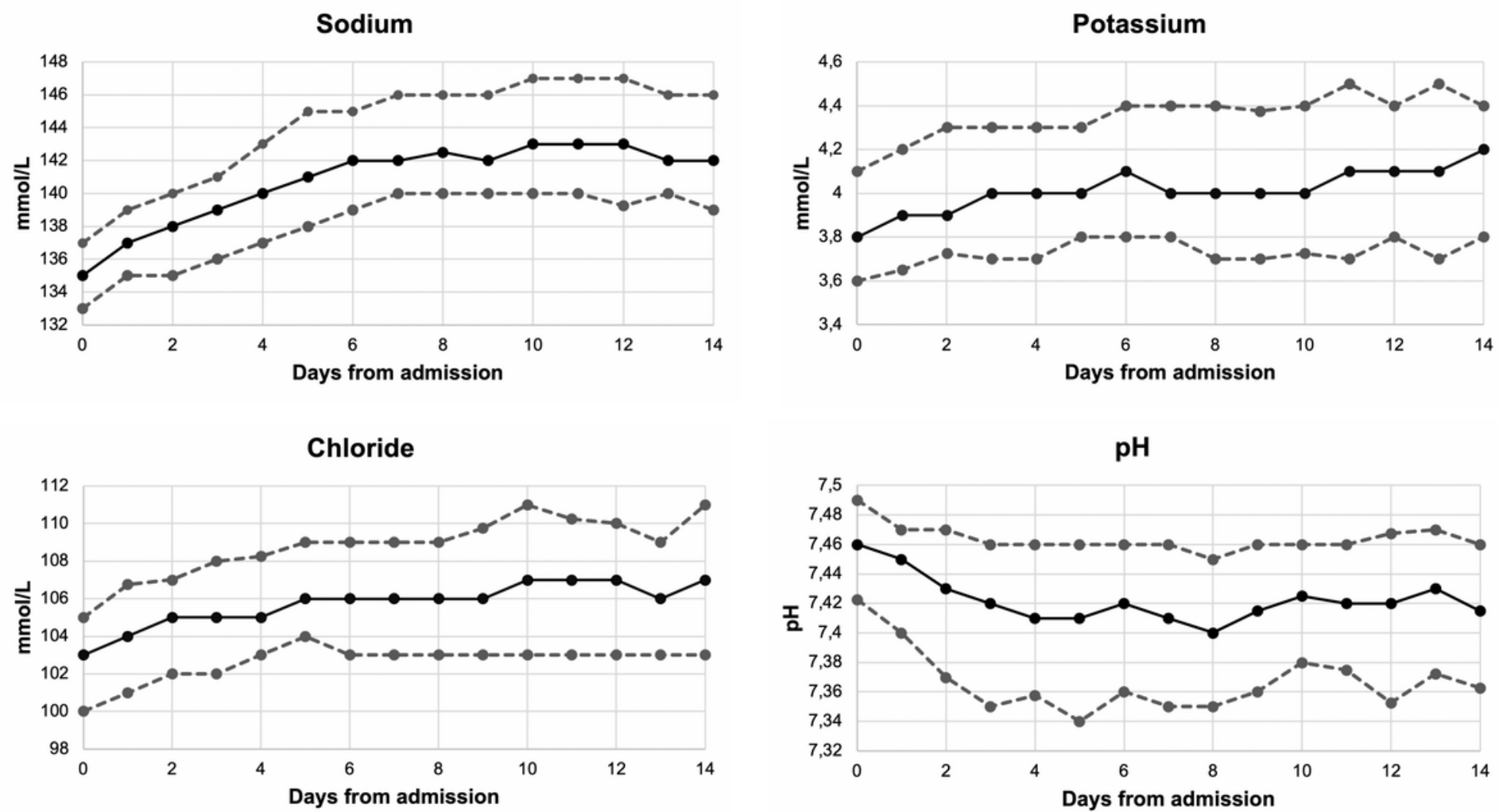

\section{Base excess}

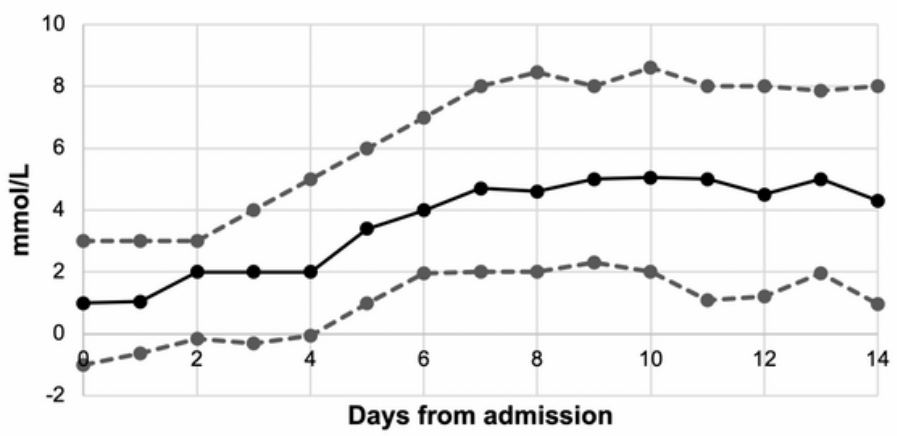

\section{Serum osmolality}

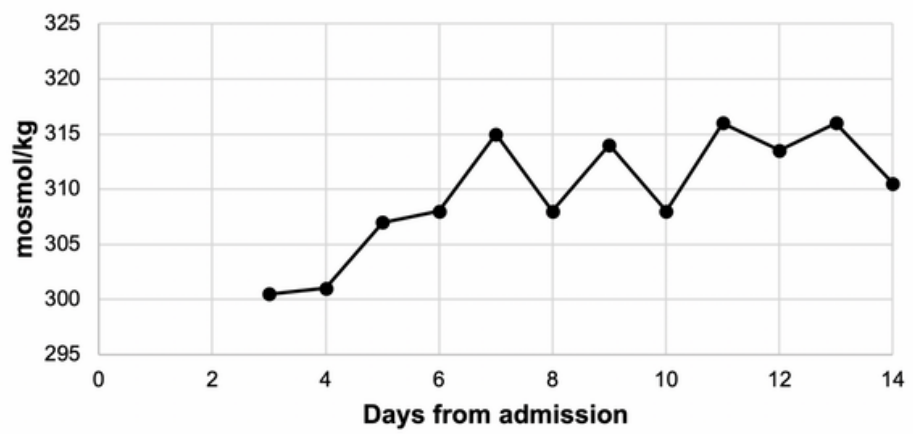


Figure 1

Dynamic pattern of laboratory parameters (sodium, potassium, chloride, $\mathrm{pH}$, base excess and serum osmolality) of 223 patients during the first two weeks of hospitalization. Data is presented as medians together with quartiles as dotted lines. Serum osmolality is showed without quartiles due to lower number of patients sampled for osmolality.


\section{Group}

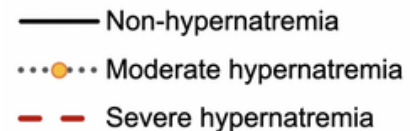

- - Severe hypernatremia

Figure 2 
Dynamic pattern of laboratory parameters (sodium, potassium, chloride, $\mathrm{pH}$ and base excess) the first two weeks of hospitalization, depending on peak level of sodium. Abbreviations: $\mathrm{NH}=$ nonhypernatremia, $\mathrm{MH}=$ moderate hypernatremia and $\mathrm{SH}$ = severe hypernatremia.

\section{Supplementary Files}

This is a list of supplementary files associated with this preprint. Click to download.

- Additionalfile1.docx

- Additionalfile2.docx

- Additionalfile3.docx 